\title{
ANTROPOMETRIA E MASTIGAÇÃO EM CRIANÇAS ASMÁTICAS
}

\section{Anthropometry and chewing in asthmatic children}

\author{
Daniele Andrade da Cunha (1), Renata Milena Freire Lima ${ }^{(2)}$, \\ Gerlane Karla Bezerra Oliveira Nascimento ${ }^{(3)}$, Renata Andrade da Cunha (4), \\ Elthon Gomes Fernandes da Silva ${ }^{(5)}$, Hilton Justino da Silva ${ }^{(6)}$, Isabella Jerônimo do Prado ${ }^{(7)}$, \\ Jabson Herber Profiro de Oliveira ${ }^{(8)}$, Sheyla de Fátima Santos Ferreira ${ }^{(9)}$, \\ Silvia Regina Arruda de Moraes ${ }^{(10)}$, Célia Maria Machado Barbosa de Castro ${ }^{(11)}$
}

\section{RESUMO}

Objetivos: caracterizar os padrões da antropometria facial em crianças asmáticas; identificar a presença de assimetrias faciais em crianças asmáticas e não asmáticas; e relacionar o lado de predomínio mastigatório com a presença de assimetria facial em crianças asmáticas e não asmáticas. Métodos: participaram da pesquisa 60 crianças com idade entre 6 e 10 anos. Destas, 30 possuíam diagnóstico em prontuário de asma moderada ou grave e 30 crianças não apresentavam asma, fazendo parte do grupo controle. Foram realizadas avaliações antropométrica facial e da mastigação dessas crianças. Resultados: em relação às mensurações antropométricas faciais nas crianças asmáticas e não-asmáticas não foram reveladas diferenças significativas entre os grupos. No que diz respeito à presença de assimetrias faciais, observou-se que estas ocorreram no grupo controle, assim como, no grupo asmático. O padrão mastigatório predominante em ambos os grupos foi o bilateral simultâneo e quando relacionados à assimetria facial e o predomínio mastigatório, não foram encontradas associações significantes. Conclusão: não foram encontradas diferenças significativas entre o grupo controle e o grupo asmático em relação às mensurações antropométricas. A assimetria facial foi observada nos dois grupos avaliados. Em ambos o padrão mastigatório bilateral simultâneo foi predominante, porém quando realizada a relação entre assimetria facial e o lado de predomínio mastigatório, não se observou relações significativas.

DESCRITORES: Antropometria; Mastigação; Asma

(1) Fonoaudióloga; Coordenadora do curso de Fonoaudiologia da Faculdade Integrada do Recife, FIR, Recife, PE; Mestre e Doutoranda em Nutrição pela Universidade Federal de Pernambuco.

(2) Fonoaudióloga do Centro Especializado em Fonoaudiologia, CEFA, Recife, PE; Especialização em Motricidade Orofacial pela Faculdade Integrada do Recife.

(3) Fonoaudióloga; Mestranda em Patologia pela Universidade Federal de Pernambuco, UFPE, Recife, PE.

(4) Fisioterapeuta; Especializanda em Fisioterapia Neurofuncional pela Faculdade Integrada do Recife.

(5) Fonoaudiólogo; Mestrando em Saúde Humana e Meio Ambiente pela Universidade Federal de Pernambuco, UFPE, Recife, PE.

(6) Fonoaudiólogo; Docente da Universidade Federal de Pernambuco, UFPE, Recife, PE; Mestre em Morfologia; Doutor em Nutrição pela Universidade Federal de Pernambuco.

(7) Nutricionista; Restaurante Bonaparte, Recife, PE.

(8) Aluno do curso de Graduação em Fonoaudiologia da Universidade Federal de Pernambuco, UFPE, Recife, PE.

(9) Aluna do curso de Graduação em Fonoaudiologia da Universidade Federal de Pernambuco, UFPE, Recife, PE.

\section{INTRODUÇÃO}

Algumas doenças crônicas, especialmente a asma, têm sido implicadas como causa de alterações nutricionais. Por ser uma doença inflamatória crônica de alta prevalência, tem sido considerada um problema de Saúde Pública, causando diversos transtornos na vida do individuo ${ }^{1}$. A maior prevalência ocorre entre as crianças ${ }^{2}$ e relaciona-se geralmente como uma das causas de baixo peso e

(10) Fisioterapeuta; Professora Adjunta da Universidade Federal de Pernambuco; UFPE, Recife, PE; Doutora em Ciências Morfofunciolnais pela Universidade de São Paulo.

(11) Médica; Professora Associada da Universidade Federal de Pernambuco, UFPE, Recife, PE; Mestre em Nutrição pela Universidade Federal de Pernambuco; Doutora em Farmacologia pela Universidade Federal do Ceará.

Conflito de interesses: inexistente 
retardo do crescimento, que pode ser iniciado pela perda de peso. Isto ocorre principalmente na asma de grau moderado e grave.

Apesar dos avanços na compreensão da sua fisiopatologia e da crescente oferta de medicações, diversos estudos vêm demonstrando aumento da prevalência, da morbidade e da mortalidade por asma em vários países ${ }^{1,3}$.

Independentemente do tratamento, ela pode repercutir sobre o crescimento, atrasando o estirão pubertário e ocorrer posteriormente, uma recuperação em relação à altura fina ${ }^{4}$. $\mathrm{O}$ crescimento de crianças com asma tem despertado interesse por ser esta uma doença crônica, e por serem utilizadas em seu manejo drogas que podem prejudicar o processo de crescimento ${ }^{1}$.

Esta doença pode afetar o crescimento facial promovendo alterações posturais da cabeça e pescoço, da colocação da língua e na posição da mandíbula ${ }^{5,6}$, além de que sua evolução crônica reflete na vida emocional, física e social da criança ${ }^{7}$.

Quando se relaciona forma e função, pode-se afirmar que, dependendo das características craniofaciais do indivíduo, a musculatura mastigatória irá sofrer adaptações compensatórias durante a realização das funções estomatognáticas ${ }^{8}$.

A realização das funções orais tais, como mastigação e deglutição, influencia e sofre influência dos aspectos morfológicos e miofuncionais, os quais possibilitam o equilíbrio e o desenvolvimento do complexo craniofacial, de modo que os distúrbios miofuncionais orais podem interferir de forma negativa no desenvolvimento craniofacial ${ }^{9}$.

Uma mastigação equilibrada deve produzir estímulos alternados nas diversas estruturas que compõem o sistema estomatognático. $O$ padrão bilateral alternado possibilita a distribuição da força mastigatória, intercalando períodos de trabalho e de equilíbrio muscular e funcional. O padrão unilateral estimula inadequadamente o crescimento ou impede a estabilização das estruturas do sistema estomatognático. O padrão unilateral proporciona uma musculatura com maior potência do lado do trabalho (lado com alimento), enquanto que a musculatura do lado do balanceio (lado sem alimento) encontra-se mais alongada e com tônus rebaixado ${ }^{10}$.

A mastigação unilateral em crianças influenciará no desenvolvimento dos terços médio e inferior da face. Crianças de 2 a 4 anos possuem condições de executar um padrão de mastigação bem próximo ao do adulto ${ }^{11}$.

Face ao exposto, acredita-se que crianças asmáticas possam apresentar assimetrias faciais, assim como alterações no padrão mastigatório quando comparadas às crianças não asmáticas. Dessa forma, a pesquisa teve como objetivos: caracterizar os padrões da antropometria facial em crianças asmáticas; identificar a presença de assimetrias faciais em crianças asmáticas e não asmáticas; e relacionar o lado de predomínio mastigatório com a presença de assimetria facial em crianças asmáticas e não asmáticas.

\section{MÉTODOS}

Trata-se de um estudo descritivo, observacional e transversal, o qual foi desenvolvido nos ambulatórios de alergologia e pediatria do Hospital das Clínicas, vinculado à Universidade Federal de Pernambuco no período de abril a dezembro de 2008.

A população alvo da presente pesquisa foi formada por dois grupos. Um composto por 30 crianças com diagnóstico em prontuário de asma moderada ou grave e o outro grupo formado por 30 crianças sem asma. A faixa etária foi entre 6 e 10 anos (pré-púbere) definida segundo a Organização Mundial de Saúde (OMS).

Somente foram incluídas no grupo de crianças controle aquelas que obedeceram aos seguintes critérios: idade entre 6 a 10 anos; não apresentar diagnóstico de asma; não fazer uso de aparelho ortodôntico; não apresentar doenças de via respiratória, assim como, características de alterações craniofaciais e/ou síndromes. Essas crianças eram atendidas no ambulatório de pediatria para realização de acompanhamento de rotina.

As crianças que formaram o grupo asmático, com faixa etária entre 6 a 10 anos, possuíam diagnóstico de asma moderada ou grave em seus prontuários e não apresentaram características de alterações craniofaciais e/ou síndromes e não usavam aparelho ortodôntico.

Os critérios de exclusão adotados nesse estudo foram: crianças que apresentassem comprometimento neurológico, crise de asma no momento da avaliação, cardiopatias graves, aparelho ortodôntico, anormalidades craniofaciais, hipertrofias de amídalas e/ou adenoides.

Buscando fazer a medição dos pontos antropométricos, a criança voluntária sentou em uma cadeira com a cabeça no plano de Frankfurt. Em seguida, a localização dos pontos para obtenção das medidas antropométricas foi marcada na face do indivíduo usando um lápis dermatográfico. E por último foi realizada e registrada em protocolo individual baseado na mensuração dos pontos: terço superior que compreende a medida entre o tragus e aglabela (tr-g); terço médio que compreende a medida entre a glabela e o subnasal ( $\mathrm{g}$-sn); terço inferior que compreende a medida do subnasal ao gnátio (sn-gn); lábio inferior que compreende 
a medida do estômio ao gnátio (sto-gn); filtro que compreende a media entre subnasal e o estômio (sn-sto); lábio superior que compreende a medida entre o subnasal e o estômio (sn-sto); Comissuras direita e esquerda que compreendem as medidas entre as comissuras labiais e os cantos dos olhos direito e esquerdo respectivamente (ex-ch) ${ }^{12}$. Com o auxílio das hastes de medição externas e de profundidade de um paquímetro digital JOMARCA ${ }^{\circledR}$ Starnieless Hardened e acurácia de 0,01mm, sem pressionar as pontas do mesmo contra a pele do voluntário. Para a avaliação da mastigação foi elaborado por uma das pesquisadoras um protocolo adaptado ${ }^{13}$. Foi solicitado que a criança comesse normalmente um pão francês de $25 \mathrm{~g}$ e durante o processo mastigatório, realizou-se a filmagem com uma filmadora digital Sony Digital Hand Cam VCR TRV 130 NTSC, fixada em um tripé com distância de um metro e meio; os dados foram gravados em fita $8 \mathrm{~mm}$.

Paralelo ao procedimento de filmagem, o tempo da mastigação foi registrado com um cronômetro da marca Casio. Após a análise das fitas, a mastigação foi caracterizada segundo quantidade de ciclos mastigatórios, tempo de mastigação e predominância por lado de mastigação.

Ressalta-se que anteriormente à coleta de dados o responsável era esclarecido sobre os objetivos da pesquisa, informado que esta foi aprovada pelo Comitê de Ética em Pesquisa da Universidade Federal de Pernambuco, sob o protocolo de número 224/2006, e em seguida solicitado que assinasse um Termo de Consentimento Livre e Esclarecido permitindo a participação da criança no estudo.

Foi realizada uma análise descritiva para expor os resultados obtidos. A apresentação das variáveis mensuradas foi feita através de tabelas incluindo também o uso de algumas medidas descritivas como mínimo, máximo, média, mediana, desvio padrão e coeficiente de variação. Para testar a suposição de normalidade das variáveis envolvidas no estudo foi aplicado o teste de Kolmogorov-Smirnov. Para análise comparativa das variáveis entre grupos foi aplicado o teste t-Student. Para a análise intragrupos foram aplicados o teste $t$-Student pareado ou ANOVA para dados pareados e para testar as diferenças encontradas pela ANOVA foi aplicado o teste LSD - (Least Stuare Difference) ${ }^{1}$. Sendo todas as conclusões tomadas ao nível de significância de $5 \%$. Para a análise das variáveis qualitativas foi aplicado o teste Qui-quadrado ou exato de Fisher, quando necessário. E por fim, para a análise de correlação foi utilizado o coeficiente de correlação linear de Pearson ${ }^{1}$. Sendo todas as conclusões tomadas ao nível de significância de 5\%.

\section{RESULTADOS}

Na Tabela 1 são descritos os dados das mensurações nas crianças asmáticas e não-asmáticas quanto a idade, terços superior, médio e inferior, gnátio, filtro, lábio superior e comissuras direita e esquerda. Nesta, houve diferenças significantes apenas para a idade, sendo o grupo asmático, em média, mais jovem que o grupo controle ( $p$-valor $=0,126)$. Os demais parâmetros mensurados não diferiram entre os dois grupos.

A Tabela 2 apresenta os dados da análise dos terços e comissuras nas crianças asmáticas e não-asmáticas. Nesta, observa-se que o grupo controle apresentou diferenças entre os terços da face. Assim, tem-se que o terço inferior da face foi significantemente maior que o terço médio ( $p$-valor=0,035). Já para o grupo asmático, as diferenças significantes foram evidenciadas para os terços e para as comissuras, nas quais o terço médio foi estatisticamente menor que o terço inferior ( $p$-valor $=0,009)$ e a comissura esquerda foi significantemente menor que à direita $(p$-valor $=0,035)$.

$\mathrm{Na}$ Tabela 3 apresenta-se a distribuição das crianças asmáticas e não-asmáticas quanto à predominância mastigatória segundo assimetria facial. A partir dos dados apresentados, pode-se dizer que não há evidências de associação entre assimetria facial e predominância mastigatória tanto nos asmáticos como nos controles, não havendo, portanto, diferenças significativas entre os resultados.

\section{DISCUSSÃO}

No que diz respeito à média de idade das crianças avaliadas neste estudo, mais especificamente ao grupo de crianças controles, que foi de 8,23 anos, observou-se que há semelhanças com a média de idade de crianças que tiveram seus pontos antropométricos faciais mensurados em pesquisa publicada, sendo esta média de 8 anos e cinco meses ${ }^{14}$.

Em relação ao terço superior da face (tr-g), foi encontrada uma média de 53,99 $\mathrm{mm}$ para o grupo de crianças controle e $53,83 \mathrm{~mm}$ para o grupo de crianças asmáticas, números que se assemelham aos dados encontrados em literatura, em que são citadas médias de $55,44 \mathrm{~mm}$ e $55,17 \mathrm{~mm}$ para essa medida $^{14,15}$.

A média encontrada para medida do terço médio da face (g-sn) foi de $53,62 \mathrm{~mm}$ no grupo controle e 52,38 no grupo asmático, estas não se distanciam dos valores encontrados em publicações, sendo este valor de $51,47 \mathrm{~mm}$ e $50,80 \mathrm{~mm}^{12,13}$.

O resultado encontrado para a medida do terço inferior da face (sn-gn) foi de $56,74 \mathrm{~mm}$ dentro do grupo controle e de 56,41 dentro do grupo 
Tabela 1 - Dados das mensurações nas crianças asmáticas e não-asmáticas quanto a idade, terços superior, médio e inferior, gnátio, filtro, lábio superior e comissuras direita e esquerda

\begin{tabular}{|c|c|c|c|c|c|c|c|c|}
\hline & $\mathbf{N}$ & Mínimo & Máximo & Média & Mediana & Desvio & C.V. & $\mathrm{p}$-valor \\
\hline \multicolumn{9}{|l|}{ Idade } \\
\hline Controle & 30 & 6 & 10 & 8,23 & 8,00 & 1,36 & 16,48 & \\
\hline Asmáticos & 30 & 6 & 10 & 7,67 & 8,00 & 1,47 & 19,17 & 0,126 \\
\hline \multicolumn{9}{|c|}{ Terço Superior } \\
\hline Controle & 30 & 44,49 & 75,71 & 53,99 & 52,97 & 6,15 & 11,39 & \\
\hline Asmático & 30 & 43,25 & 68,81 & 53,83 & 52,13 & 6,24 & 11,58 & 0,923 \\
\hline \multicolumn{9}{|c|}{ Terço Médio } \\
\hline Controle & 30 & 40,22 & 61,75 & 53,62 & 54,82 & 4,87 & 9,08 & \\
\hline Asmático & 30 & 46,29 & 61,81 & 52,38 & 52,17 & 3,88 & 7,41 & 0,283 \\
\hline \multicolumn{9}{|c|}{ Terço Inferior } \\
\hline Controle & 30 & 45,90 & 69,09 & 56,74 & 57,36 & 5,25 & 9,25 & \\
\hline Asmático & 30 & 42,29 & 74,77 & 56,41 & 55,47 & 6,39 & 11,33 & 0,827 \\
\hline \multicolumn{9}{|l|}{ Gnátio } \\
\hline Controle & 30 & 29,83 & 46,31 & 38,34 & 38,08 & 3,90 & 10,18 & \\
\hline Asmático & 30 & 24,97 & 52,05 & 36,43 & 36,43 & 5,41 & 14,69 & 0,224 \\
\hline \multicolumn{9}{|l|}{ Filtro } \\
\hline Controle & 30 & 8,22 & 18,78 & 12,43 & 11,86 & 2,67 & 21,47 & \\
\hline Asmático & 30 & 8,72 & 17,43 & 12,46 & 12,46 & 2,20 & 17,63 & 0,960 \\
\hline \multicolumn{9}{|c|}{ Lábio Superior } \\
\hline Controle & 30 & 12,79 & 23,17 & 17,72 & 17,24 & 2,92 & 16,46 & \\
\hline Asmático & 30 & 11,16 & 28,58 & 17,51 & 16,09 & 4,18 & 23,90 & 0,820 \\
\hline \multicolumn{9}{|c|}{ Comissura Direita } \\
\hline Controle & 30 & 56,07 & 73,09 & 65,34 & 65,20 & 4,15 & 6,36 & \\
\hline Asmático & 30 & 53,39 & 77,70 & 64,45 & 64,58 & 4,83 & 7,49 & 0,513 \\
\hline \multicolumn{9}{|c|}{ Comissura Esquerda } \\
\hline Controle & 30 & 59,10 & 71,65 & 65,12 & 64,84 & 3,59 & 5,51 & \\
\hline Asmático & 30 & 48,69 & 74,88 & 63,40 & 63,29 & 5,63 & 8,88 & 0,163 \\
\hline
\end{tabular}

asmático essas medidas também não diferem estatisticamente do valor encontrado em outro estudo que traz o valor médio de $58,20 \mathrm{~mm}$ para essa medida ${ }^{14}$

Com relação à medida antropométrica do gnátio (sto-gn) foi observado que o grupo controle apresentou uma média de $38,34 \mathrm{~mm}$ e o grupo asmático $36,85 \mathrm{~mm}$. Comparando-se com os achados percebe-se que estes valores se equiparam com os encontrados em literatura que referem uma média de $39,70 \mathrm{~mm}$ e $39,17 \mathrm{~mm}$ para essa medida ${ }^{14,15}$.

Ainda no que diz respeito à medida do gnátio, foi visto que as crianças asmáticas apresentaram um gnátio discretamente menor em comparação ao grupo controle, corroborando os achados do estudo em que foram avaliadas cefalometricamente a morfologia craniofacial e postura da cabeça de
100 crianças com e sem asma, dos 6 aos 16 anos e observaram que as asmáticas apresentaram tendência de desenvolvimento de retrognatismo maxilo-mandibular, além das relações dentoalveolares estarem alteradas ${ }^{16}$.

A média encontrada para medida do filtro (sn-ls) foi de $12,43 \mathrm{~mm}$ no grupo controle e 12,46 no grupo asmático. Pode-se dizer que estas se mostram de acordo com as médias $13,12 \mathrm{~mm}$ e 13,05 encontradas em outros estudos ${ }^{12,13}$.

No que diz respeito à média antropométrica do lábio superior (sn-sto) foram encontrados os valores de $17,72 \mathrm{~mm}$ para as crianças do grupo controle e $17,51 \mathrm{~mm}$ para as crianças do grupo de asmáticos. Valores semelhantes aos encontrados em outros trabalhos, que foram de $18,12 \mathrm{~mm}$ e $18,03 \mathrm{~mm}^{14,15}$. 
Tabela 2 - Dados da análise dos terços e comissuras nas crianças asmáticas e não-asmáticas

\begin{tabular}{|c|c|c|c|c|c|c|c|c|c|}
\hline & $\mathbf{N}$ & Mínimo & Máximo & Média & Mediana & Desvio & C.V. & p-valor & Sig. \\
\hline \multicolumn{10}{|l|}{ Controle } \\
\hline \multicolumn{10}{|l|}{ Terço } \\
\hline Superior ${ }^{(1)}$ & 30 & 44,49 & 75,71 & 53,99 & 52,97 & 6,15 & 11,39 & & \\
\hline Médio ${ }^{(2)}$ & 30 & 40,22 & 61,75 & 53,62 & 54,82 & 4,87 & 9,08 & & $2 \times 3$ \\
\hline Inferior ${ }^{(3)}$ & 30 & 45,90 & 69,09 & 56,74 & 57,36 & 5,25 & 9,25 & 0,035 & \\
\hline \multicolumn{10}{|l|}{ Comissura } \\
\hline Direita & 30 & 56,07 & 73,09 & 65,34 & 65,20 & 4,15 & 6,36 & & \\
\hline Esquerda & 30 & 59,1 & 71,65 & 65,12 & 64,84 & 3,59 & 5,51 & 0,720 & \\
\hline \multicolumn{10}{|l|}{ Asmáticos } \\
\hline \multicolumn{10}{|l|}{ Terço } \\
\hline Superior ${ }^{(1)}$ & 30 & 43,25 & 68,81 & 53,83 & 53,13 & 6,24 & 11,58 & & \\
\hline Médio ${ }^{(2)}$ & 30 & 46,29 & 61,81 & 52,38 & 52,17 & 3,88 & 7,41 & & $2 \times 3$ \\
\hline Inferior ${ }^{(3)}$ & 30 & 42,29 & 74,77 & 56,41 & 55,47 & 6,39 & 11,33 & 0,009 & \\
\hline \multicolumn{10}{|l|}{ Comissura } \\
\hline Direita & 30 & 53,39 & 77,70 & 64,58 & 64,07 & 4,83 & 7,49 & & \\
\hline Esquerda & 30 & 48,69 & 74,88 & 63,40 & 63,29 & 5,63 & 8,88 & 0,035 & \\
\hline
\end{tabular}

Tabela 3 - Distribuição das crianças asmáticas e não-asmáticas quanto a predominância mastigatória segundo assimetria facial

\begin{tabular}{|c|c|c|c|c|c|}
\hline \multirow{3}{*}{ Predominância Mastigatória } & \multicolumn{4}{|c|}{ Assimetria Facial } & \multirow{3}{*}{ p-valor } \\
\hline & \multicolumn{2}{|c|}{ Direito } & \multicolumn{2}{|c|}{ Esquerdo } & \\
\hline & $\mathbf{N}$ & $\%$ & $\mathbf{N}$ & $\%$ & \\
\hline \multicolumn{6}{|l|}{ Controle } \\
\hline Lado direito & 3 & 20,0 & 4 & 26,7 & \\
\hline Lado esquerdo & 4 & 26,7 & 2 & 13,3 & \\
\hline Bilateral simultâneo & 8 & 53,3 & 9 & 60,0 & 0,781 \\
\hline \multicolumn{6}{|l|}{ Asmáticos } \\
\hline Lado direito & 4 & 23,5 & 2 & 15,4 & \\
\hline Lado esquerdo & 4 & 23,5 & 4 & 30,8 & \\
\hline Bilateral simultâneo & 9 & 52,9 & 7 & 53,8 & 0,893 \\
\hline
\end{tabular}

A média de valores encontrados na mensuração da medida da comissura direita (ex-ch) foi de 65,34 $\mathrm{mm}$ para o grupo controle e $64,58 \mathrm{~mm}$ para o grupo asmático. Comparando-se às médias descritas em literatura, percebe-se que estes valores foram maiores do que os encontrados, sendo relatada uma média de $62,21 \mathrm{~mm}$ e $62,68 \mathrm{~mm}$ para essa medida ${ }^{14,15}$.

Com relação à medida antropométrica da comissura esquerda (ex-ch) a média de valores encontrados foram de $65,12 \mathrm{~mm}$ para o grupo controle e $63,40 \mathrm{~mm}$ para o grupo asmático. Dessa forma, percebe-se que o grupo de crianças asmáticas apresentou de forma discreta uma medida menor que as crianças do grupo controle. Comparando-se às médias descritas em literatura, mais uma vez percebe-se que estes valores foram maiores do que os encontrados sendo relatada uma média de $61,40 \mathrm{~mm}$ e $61,89 \mathrm{~mm}$ para essa medida ${ }^{14,15}$.

No que diz respeito às assimetrias faciais das crianças do grupo controle e das crianças do grupo asmático, foi visto que no grupo controle há diferença entre os terços da face, sendo o terço inferior significativamente maior que os terços superior e médio, dados que diferem dos encontrados em 
outro estudo, em que as diferenças encontradas entre os terços não foram significantes ${ }^{14}$.

Já para o grupo asmático verificou-se diferenças significantes para os terços e para as comissuras. O terço médio foi estatisticamente menor que o terço inferior e a comissura esquerda foi significativamente menor que à direita. Em estudo publicado foi relatado que a média do lado esquerdo das crianças pesquisadas também se mostrou estatisticamente menor que a média encontrada do lado direito ${ }^{14,15}$.

Quando se relacionou a predominância mastigatória e a assimetria facial não foram encontradas diferenças significantes. Entretanto, é possível observar que das 30 crianças controles que apresentaram assimetria facial, pelo mesmo 17 apresentaram um padrão bilateral simultâneo. De forma bastante semelhante ocorreu com as crianças asmáticas, das 30 avaliadas e que apresentaram assimetria facial, 16 tiveram como padrão mastigatório o bilateral simultâneo. O que reforça as informações encontradas em literatura que afirmam que crianças com alterações do padrão respiratório desenvolvem a estrutura mandibular de forma mais lenta e apresentam um padrão mastigatório pouco eficiente e consequentemente a musculatura facial se encontra com tônus rebaixado ${ }^{10,17}$.

Em pesquisa realizada com 77 crianças respiradoras orais e 154 respiradoras nasais entre seis e dez anos na qual um dos objetivos era pesquisar a função mastigatória em crianças respiradoras orais, foi observado que as crianças respiradoras orais apresentaram alterações no padrão mastigatório, quando comparados aos respiradores nasais. Nesse estudo não houve diferença significante nos padrões de lateralidade da mastigação, porém, a mastigação bilateral alternada ocorreu em apenas pouco mais da metade das crianças respiradoras orais ${ }^{18}$. Esse padrão mastigatório depende da adequação do crescimento e do desenvolvimento craniofacial, do equilíbrio oclusal e da maturação neuromuscular. Crianças com alterações na respiração geralmente não apresentam equilíbrio morfofuncional, o que pode justificar as alterações da mastigação ${ }^{10}$.

\section{CONCLUSÃO}

De acordo com os dados observados têm-se que:

1. Ocorreram diferenças significantes apenas para a idade, sendo o grupo asmático, em média, mais jovem que o grupo controle, e as demais medidas avaliadas não diferiram entre os dois grupos.

2. O grupo controle apresentou diferenças entre os terços da face, sendo o terço inferior significantemente maior que os terços superior e médio respectivamente. Já no grupo asmático verificou-se que o terço médio foi estatisticamente menor que o terço inferior e a comissura esquerda foi significativamente menor que à direita, podendo sugerir uma maior presença de assimetria facial nas crianças que apresentam asma.

3. Não foram encontradas diferenças significantes quando relacionado à predominância mastigatória e à assimetria facial. Mas, observa-se como as crianças avaliadas, tanto as do grupo controle, como as do grupo asmático apresentam alterações em seu padrão mastigatório, sendo este predominantemente bilateral simultâneo.

\section{AGRADECIMENTOS}

Ao CNPq - Edital Universal 15/2007. 


\begin{abstract}
Purposes: to characterize the facial anthropometry patterns in asthmatic children; to identify the presence of facial asymmetry and to relate the prevalence of masticatory side with the presence of facial asymmetry in asthmatic and non-asthmatic children. Methods: 60 children aged 6 to 10 years were evaluated. Thirty among them had a diagnosis of moderate or severe asthma and 30 children with no symptoms were the control group based on hospital's records. Facial anthropometric and chewing evaluations were carried through in these children. Results: for facial anthropometric measurements in asthmatic and non-asthmatics children there were no significant differences between the groups but the finding of facial asymmetry was observed in both groups. The predominant masticatory pattern in both groups was the simultaneous bilateral and when it was related to facial asymmetry and masticatory predominance, there were no significant associations. Conclusion: no significant differences were found between the control and asthmatic group as for anthropometric measurements, however, the facial asymmetry was observed in the two groups. The simultaneous bilateral masticatory pattern was predominant in both groups, but the relation between facial asymmetry and the predominance of masticatory side were not significant.
\end{abstract}

KEYWORDS: Anthropometry; Mastication; Asthma

\section{REFERÊNCIAS}

1. Bozkurt B, Karakaya G, Kalyoncu AF. Seasonal rhinitis, clinical characteristics and risk factors for asthma. Int Arch Allerg Immunol 2005; 138(1):73-9. 2. McFadden ER, Gilbert IA. Asthma. New Eng J Medic. 1992; 327(27):1928-37.

3. Burney PG, Chinns S, Rona RJ. Has the prevalence of asthma increased in children? Evidence from the national study of health and growth. Brit Med J. 1990; 300(6735):1973-86

4. Preece MA, Law CM, Davies PSW. The growth of children with chronic pediatric disease. Clin Endocrinol Metabol. 1986; 15:453-77.

5. Passàli D, Damiani V, Passàli FM, Passàli GC, Bellussi L. Atomized nasal douche vs nasal lavage in acute viral rhinitis. Arch Otolaryngol Head Neck Surg. 2005; 131(9):788-90.

6. Fernandes ALG. Asma, uma doença crônica cujas manifestações vão além do desconforto respiratório, limitação física e redução da qualidade de vida: [editorial]. J Bras Pneumol. 2009; 35(4):293-4.

7. Campanha SMA, Freire LMS, Fontes MJF. O impacto da asma, da rinite alérgica e da respiraçã o oral na qualidade de vida de crianças e adolescentes. Rev. CEFAC. 2008; 10(4):513-9. dx.doi.org/10.1590/S1516-18462008000400011

8. Rodrigues KA, Rahal AA. Influência da tipologia facial na atividade eletromiográfica do músculo masseter durante o apertamento dental em máxima intercuspidação. Rev. CEFAC. 2003; 5(2):127-30.

9. Merigui LBM. Características miofuncionais orofaciais e vocais das crianças do município de Monte Negro/RO. [dissertação] Bauru (SP): Universidade de São Paulo; 2006.

10. Bianchini EMG. Descrição da tipologia facial e relação póstero-anterior das bases ósseas. In: Marchesan IQ. Fundamentos em fonoaudiologia: aspectos clínicos da motricidade oral. Rio de Janeiro: Guanabara-Koogan; 1998. p. 46-7.

11. Amaral BD. Mastigação unilateral $X$ oclusão normal: um estudo sobre sua ocorrência em crianças de 4 a 5 anos. Rev. CEFAC. 2000; 2(1):23-30.

12. Cattoni DM, Fernandes FDM, Andrade CRF, Latorre MRDO. Estudo sobre as medidas faciais em crianças: correlações com alteração de mordida e uso de aparelho ortodôntico. Rev Soc Bras Fonoaudiol. 2005; 10(1):1-6.

13. Marchesan IQ, Cattoni DM, Fernandez FDM, Latorre MRDO. Medidas antropométricas faciais em crianças segundo períodos da dentição mista. Rev. CEFAC. 2003; 5(1):21-9.

14. Cattoni DM, Fernandes FDM, Di Francesco RC, Latorre MRDO, Medidas e proporções antropométricas orofaciais de crianças respiradoras orais. Rev Soc Bras Fonoaudiol. 2008; 13(2):119-26.

15. Cattoni DM, Fernandes FDM. Medidas e proporções faciais em crianças: contribuições para a avaliação miofuncional orofacial. Pró-Fono. 2004; 16(1):7-18.

16. Wenzel A, Höjensgaard E, Henriksen J. Craniofacial morphology and head posture in children with asthma and perennial rhinits. Eur $\mathrm{J}$ Orthodont. 1985; 7(2):83-92. 
17. Shikata N, Ueda HM, Kato M, Tabe H, Nagaoka $\mathrm{K}$, Nakashima $\mathrm{Y}$, et al. Association between nasal respiratory obstruction and vertical mandibular position. J Oral Rehabil. 2004; 31(10):957-62.
18. Cunha DA, Silva GAP, Motta MEFA, Lima CR, Silva HJ. A respiração oral em crianças e suas repercussões no estado nutricional. Rev. CEFAC. 2007; 9(1):47-54. dx.doi.org/10.1590/ S1516-18462007000100007

RECEBIDO EM: 17/03/2009

ACEITO EM: 03/09/2009

Endereço para correspondência:

Hilton Justino da Silva

Rua São Salvador, 105 /1002

Recife - PE

CEP: $52020-200$

E-mail: hiltonfono@ @otmail.com

Rev CEFAC, v.11, Supl3, 341-348, 2009 